\title{
Fiber optic civil structure monitoring system
}

\author{
Jose Miguel López-Higuera \\ César Jauregui Misas \\ Antonio Quintela Incera \\ Juan Echevarría Cuenca \\ Universidad de Cantabria \\ Grupo de Ingeniería Fotónica \\ E.T.S.I.I. y Telecomunicación \\ Dpto. TEISA \\ Avda. Los Castros s/n-c.p. 39005 \\ Santander \\ Spain \\ E-mail: higuera@teisa.unican.es
}

\begin{abstract}
A civil structure monitoring system based on fiber Bragg grating technology is presented. A complete set of new transducers, both for concrete and steel monitoring, is designed, fabricated, and successfully checked both in laboratory and in-field conditions. (c) 2005 Society of PhotoOptical Instrumentation Engineers. [DOI: 10.1117/1.1882392]
\end{abstract}

Subject terms: fiber Bragg grating; civil structure monitoring; fiber optic transducer; fiber optic sensors; quasi-distributed sensing.

Paper 040081 received Feb. 16, 2004; revised manuscript received Jul. 16, 2004; accepted for publication Nov. 4, 2004; published online Mar. 30, 2005.

\section{Introduction}

Currently, civil engineering is demanding new ways of monitoring structures. Such an interest is based on the fact that civil structures and their maintenance are extremely expensive. The high costs of maintenance are due to the fact that the monitoring methods used today detect the structure's degradation when it is too late, that is, when physical damage has already occurred. This monitoring is mainly based on periodical visual inspections that try to detect cracks or other forms of physical degradation in the structure. However, civil engineers have recently realized that this kind of monitoring is not enough, since most of the time it leads to expensive reparation works. Thus, the idea of inner-monitoring of the structure reveals itself as a much more useful technique for the early detection of structural degradation.

This inner-monitoring demands embeddable transducers able to withstand a harsh operating environment during the entire lifetime of the structure. Besides, low size is a key feature to altering the measurement point as little as possible. Conventional measurement technologies such as resistive strain gauges are not appropriate for this task. In general, they have not been designed for being embedded because, although they are small, they tend to be fragile and the resistive coils can be quite exposed. Moreover, the operating life of these devices does not exceed one year, which invalidates them for long-term monitoring. Also, having an electrical current flowing inside the concrete accelerates its degradation. On the other hand, fiber optic sensing technology ${ }^{1,2}$ gathers all the desirable characteristics that have been mentioned before, plus several more: dielectric nature, compatibility with civil structures' materials, low weight, etc. Among all the possible fiber optic transducers, there are two that are especially suitable for civil engineering: those based on white light interferometry ${ }^{3,4}$ and those based on fiber Bragg grating (FBG) technology. ${ }^{5,6}$ The latter offers several advantages of its own that make them very attractive: possibility of multiplexing, i.e., arranging several transducers along the same fiber; wavelength encoding of the measured information; and capability of simultaneously monitoring both strain and temperature. The first of these advantages brings a significant reduction on sensor unitary costs, as several of them

0091-3286/2005/\$22.00 @ 2005 SPIE can share the same optical channel. The second advantage gives robustness to the measurement against interference, because it is encoded in an absolute parameter.

In this work, the whole process of monitoring a civil structure with custom-designed transducers is described. This process comprises the design of the different transducers, the laboratory tests, real in-field installation, and field tests. In the framework of the project described here, two main types of transducers were designed, tested, and installed. There were ones designed to be embedded in concrete, and others devoted to the measurement of strain and temperature in iron structures (meant to be fixed at their surface).

The work is organized as follows: in Sec. 2, the design of the different transducers is described; in Sec. 3, the laboratory tests and their results are examined; in the following section the in-field installations in two different bridges are described and the results of the tests performed on the measurement system are shown; finally, several conclusions are presented.

\section{Design of the Transducer}

The two transducers developed in the project are described in this section: one designed to be embedded in concrete structures and the other thought to replace traditional resistive strain gauges. The latter were designed to be as similar as possible to these traditional sensing devices.

All these transducers are based on FBGs. However, a drawback of this technology is that it is simultaneously sensitive to both temperature and strain. This fact can lead to the misinterpretation of a wavelength shift to be strain driven when it is actually temperature driven. Therefore, each transducer should incorporate a way of discriminating between these two parameters if accurate measurements are required. $^{7}$ There are several discrimination techniques available ${ }^{8-11}$ that could be used in the transducers. The one selected in this case uses two FBGs at different Bragg wavelengths. One of them must be strain isolated and, thus, only temperature sensitive. ${ }^{12}$ This technique is, at the same time, the simplest and most widespread of all.

\subsection{Embeddable Transducers}

This transducer was designed to be directly embedded in concrete without further protection. This implies that its body should be hard enough to protect the fiber from con- 


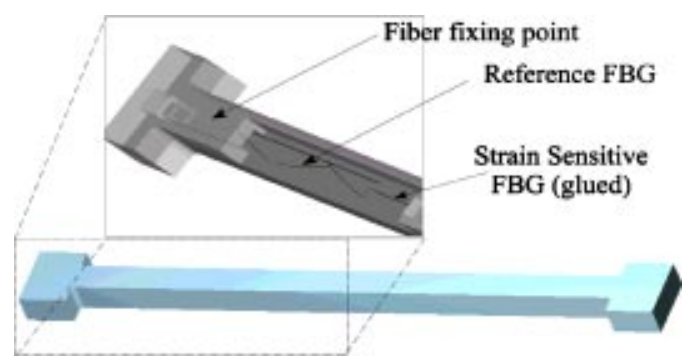

Fig. 1 Diagram showing the inner structure of the embeddable transducer.

crete. Besides, as the transducer's body is going to be in direct contact with concrete, it must be chemically and mechanically as compatible as possible with it. It must be taken into account that concrete is an alkaline medium that may attack some materials in long-term operations. This is why special attention should be devoted to the choice of the material for the transducer's body. In this particular case, a plastic material known as polymethylmethacrylate (PMMA) was chosen because of its high compatibility with concrete.

As has already been said, two FBGs were employed to perform strain-temperature discrimination. One of them must be strain-isolated, serving as a temperature reference. The strain-sensitive FBG was completely glued to the body of the transducer, while the temperature reference one was let loose. The latter implies that the strain-sensitive grating was completely covered with glue in such a way that it was embedded in a small channel prepared at the center of the transducer, as seen in Fig. 1. Therefore, the fiber inside the transducer was only fixed at three points: the two extremes of the transducer and the strain-sensitive grating. This fact allowed the loosening of the reference grating. It was introduced in a 5-mm-diam tube, which was placed between the strain FBG and one of the extremes, and was helically coiled in the inside, as schematically shown in Fig. 1. Therefore, the transducer was able to elongate without straining the reference FBG. This is because the length of fiber contained in the tube was substantially longer than the length of it.

The transducer was capped at both extremes for avoiding the concrete reaching the sensing and reference FBGs placed inside it. Moreover, these caps also serve as the fiber's fixing points located at the ends of the transducer.

One important detail that will ultimately affect the accuracy of the measurements is the type and quality of the glue. It must guarantee a perfect strain transference from the body to the sensing FBG. Moreover, these straintransference properties must remain unchanged for a wide range of environmental conditions, i.e., temperature and humidity levels. Therefore, thorough experimental works that included environmental tests were carried out to select the proper glue. Finally, a cianocrylate-based glue, specifically designed for traditional resistive strain gauges, was chosen.

This transducer was meant to directly measure the elongation of concrete. Therefore, the transducer had to be provided with some means of anchoring to it. In this particular case, as shown in Fig. 1, the 43-cm-long body of the transducer was ended with two wider heads that served as anchors. Thus, when the concrete solidifies, it will stretch or compress the transducer from these heads.

To check the quality of the design and the appropriateness of the material chosen to make it, a calibration test was carried out. Since all the transducers used in the works reported in this research were hand-assembled, it was necessary to perform this calibration test on all of them. This way the gauge factor (conversion factor between wavelength shift and strain) could be determined for each transducer. This test consisted in applying a $0.3-\mathrm{mm}$ linear elongation to the transducers by stretching them from their heads. This task was done by employing a pulling machine used in material science laboratories. An example of the results obtained with this calibration test can be seen in Fig. 2. There the wavelengths of the two FBGs are plotted versus the force applied to the transducer (magnitude directly proportional to the elongation). It can be seen that only the

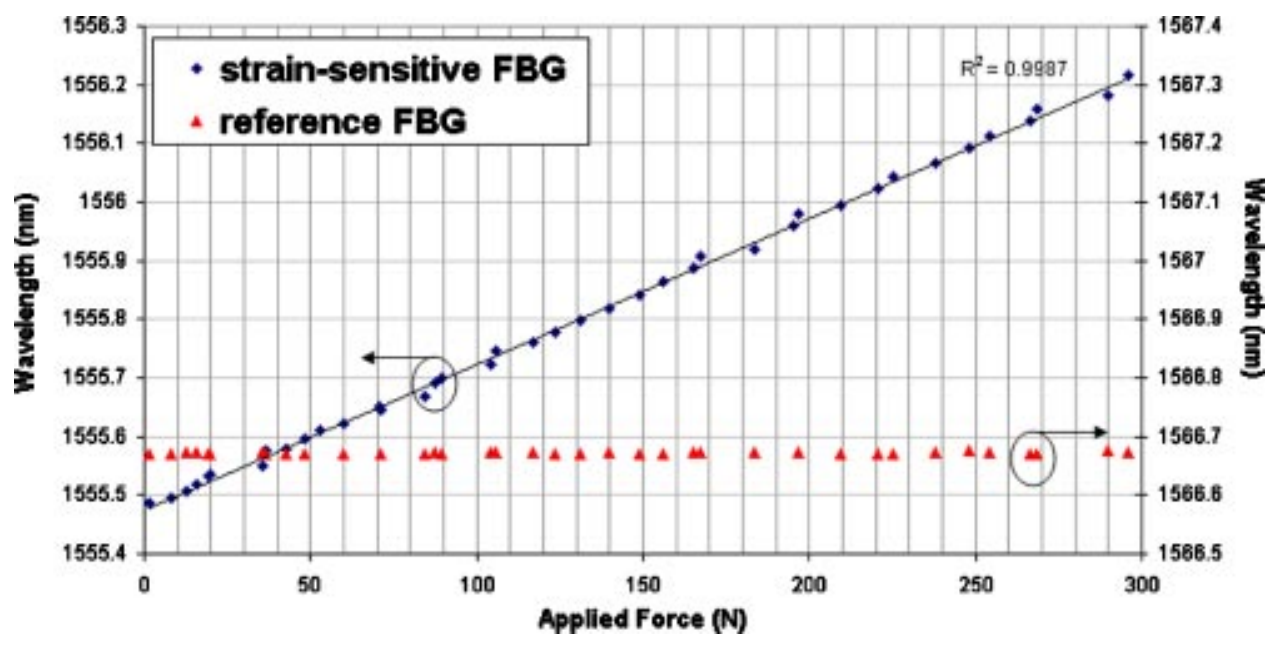

Fig. 2 Results of the calibration test applied to one of the embeddable transducers. The responses of the strain-sensitive FBG (diamonds) and the reference FBG (triangles) are shown. The linear tendency of the strain-sensitive FBG response is also presented. 


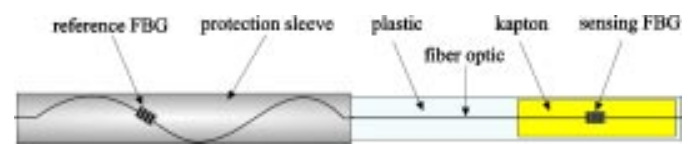

Fig. 3 Diagram of the gauge-like transducer.

wavelength of the strain-sensitive (fixed) FBG shifted. Meanwhile, that of the reference FBG remained unchanged. The linear tendency of the data corresponding to the strain-sensitive FBG has also been provided in this graph. It can be seen that the linearity of the response was fairly good.

Up until now, just the embeddable transducers have been described. However, it should also be mentioned that the optical channel that linked them had also to be properly shielded to withstand the hard operating conditions of a real installation. A custom fiber channel was developed for this purpose in collaboration with Alcatel España.

\subsection{Gauge-Like Transducers}

The main objective of these transducers was to measure strain and temperature on the surface of concrete and steel structures.

As it has already been mentioned, these transducers were designed to be as similar as possible (in size and shape) to traditional resistive strain gauges that are commonly used in civil engineering. There are two main reasons for this decision: first of all, with this strategy the technology-change shock for civil engineers would be considerably reduced, and the transducers would benefit from the ample know-how of installation, manipulation, adhesives, etc. that already exists for strain gauges.

Fiber optics is a rather fragile medium and, therefore, must be protected from external aggressions. This is especially true for surface transducers. However, the required low size and thickness of these transducers introduce a limitation in the protection level that can be provided to them. This implies that, after the installation of the transducer, an extra protection will be required. In the case of the transducers described in this section, the first-level protection was a plastic sheet in which the fiber was embedded. However, in the surroundings of the grating, this plastic material was replaced by two foils of Kapton ${ }^{\circledR}$ that covered the fiber completely, as seen in Fig. 3. This material is also the one used in traditional resistive strain gauges, and so, the same adhesives developed for them can be used with this FBG transducer. Kapton ${ }^{\circledR}$ has the additional advantages of having an extremely small expansion coefficient and ensuring a good strain transfer to the grating.

Also in this case, the inclusion of an extra FBG that acts as a temperature reference is required. This grating was placed inside a rigid protection tube as depicted in Fig. 3. The mission of this tube was to protect the grating and to procure enough room to coil it. This tube also served as a transition between the flat part of the transducer (that comprises the plastic and Kapton ${ }^{\circledR}$ protection) and the optical channel.

\section{Laboratory Demonstration}

A laboratory demonstration was set up to check the actual performance of the two types of transducers. It consisted of heavily monitoring a $0.2 \times 0.3 \times 5.6-\mathrm{m}$ concrete beam, shown in Fig. 4(a), and was planned to cover all the possible situations for the transducers. Thus, the distribution of the transducers, shown in Fig. 4(b), was decided. It involved both embeddable and gauge-like transducers. The former were directly embedded in concrete (transducers A, $\mathrm{A}^{\prime}, \mathrm{D}, \mathrm{D}^{\prime}, \mathrm{E}$ and $\mathrm{E}^{\prime}$ ) by attaching them to the iron skeleton of the beam (armor). On the other hand, the gauge-like transducers were used to monitor the behavior of the surface of the beam at the top (transducers $\mathrm{H}$ and $\mathrm{H}^{\prime}$ ) and bottom sides $\left(\mathrm{G}\right.$ and $\left.\mathrm{G}^{\prime}\right)$, and the deformation of the iron armor (transducers B, B', C, $\mathrm{C}^{\prime}, \mathrm{F}$ and $\mathrm{F}^{\prime}$ ). Although these gauge-like transducers were not specifically designed to be embedded, they are embeddable if proper protection is provided, just like traditional strain gauges. Therefore, the proposed distribution of transducers was able to check the ability of these sensors to monitor different materials.

Once the transducers were attached to the armor of the beam, the concrete was poured down and all the sensors were embedded. From this moment on, the solidifying process of the beam was monitored. The chemical reactions leading to the hardening of the concrete are very exothermic, as clearly shown in Fig. 5. The graph represents the temperature evolution of the concrete for a 12-day period. The experiment was carried out during cold winter days that dropped the room temperature down to $10^{\circ} \mathrm{C}$. As can be seen, the concrete overheating due to the chemical reactions reached a maximum of around $12^{\circ}$ above room temperature. On the other hand, the graph also shows that the solidifying process lasted about $96 \mathrm{~h}$. All the ripples that appear beyond that time are due to day-night cycles.

Once this temperature monitoring was carried out and after the concrete had hardened, the beam was subjected to a load test. During it, an increasing load was applied to the beam until it cracked. This load was simultaneously applied at two points in the central upper part of the beam. These two points were $1 \mathrm{~m}$ away from each other. Figure 6 shows the response of the gauge-like transducer B to the increasing load. Since this was a bottom transducer and the load was applied at the upper part of the beam, the transducer was stretched (positive strain), as indicated by the graphs. Figure 6 includes two graphs that are two different representations of the same information. Figure 6(a) shows the time evolution of the deformation of the beam as measured by a FBG transducer and a conventional strain gauge. It can be seen that the responses of these transducers were quite similar both in shape and in measured strain. The latter is true even though the applied deformation was not linear. Instead, the load test included first a progressive load up to a maximum of around $1000 \mu \epsilon$. This maximum load was held for around $10 \mathrm{~min}$, and then it was released. Later on the beam was loaded again. This time the applied load was continuously increased until the beam cracked. All of these were fairly reproduced by both the FBG and the conventional transducers. On the other hand, although Fig. 6(a) is a good representation for evaluating the temporal response of the transducers, it is not the best for carrying out a comparison between them. That is why Fig. 6(b) has been included. There, the measured strain versus the applied load 
(a)
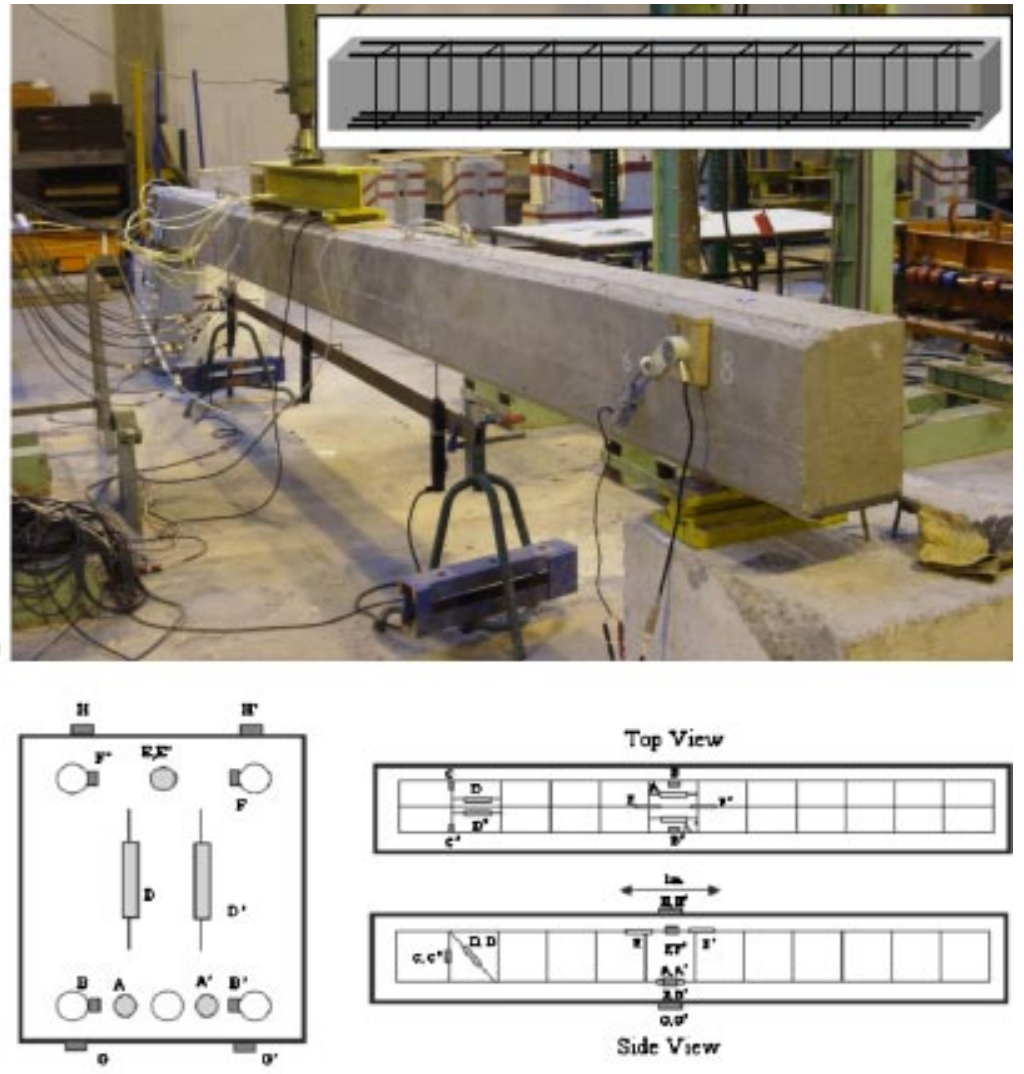

Front View

Embeddable Transducer

(b)

External Gaug-itke Transciucer
Enbedded Gauge-hke Transducer

Zmbeddable Transatucer

Fig. 4 (a) Monitored concrete beam during the load test. The inset shows a 3-D view of the iron armor of the beam. (b) Distribution of transducers inside the concrete beam used for the laboratory demonstration.

is plotted for both transducers. For the sake of clearness, only the data of the second load have been represented. Once again it can clearly be seen that the responses of both sensors were very similar. It can also be observed that these responses were very linear up to 11 tons of applied load. This is because below that point concrete works in its elas- tic zone, which is characterized by a linear relationship between load and elongation. However, from that point on the graph is no longer linear: for a constant increment of applied load, the elongation rate grows more and more. This is the typical behavior of concrete in its plastic zone, and it predicts the collapse of the beam. It is also in these

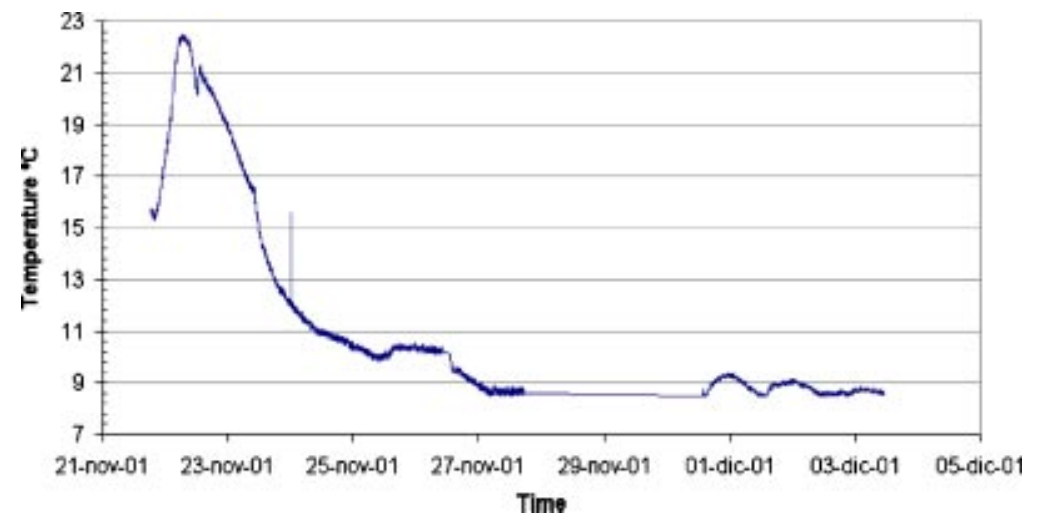

Fig. 5 Temperature evolution of the concrete during its solidifying process as recorded by transducer $\mathrm{E}^{\prime}$. 

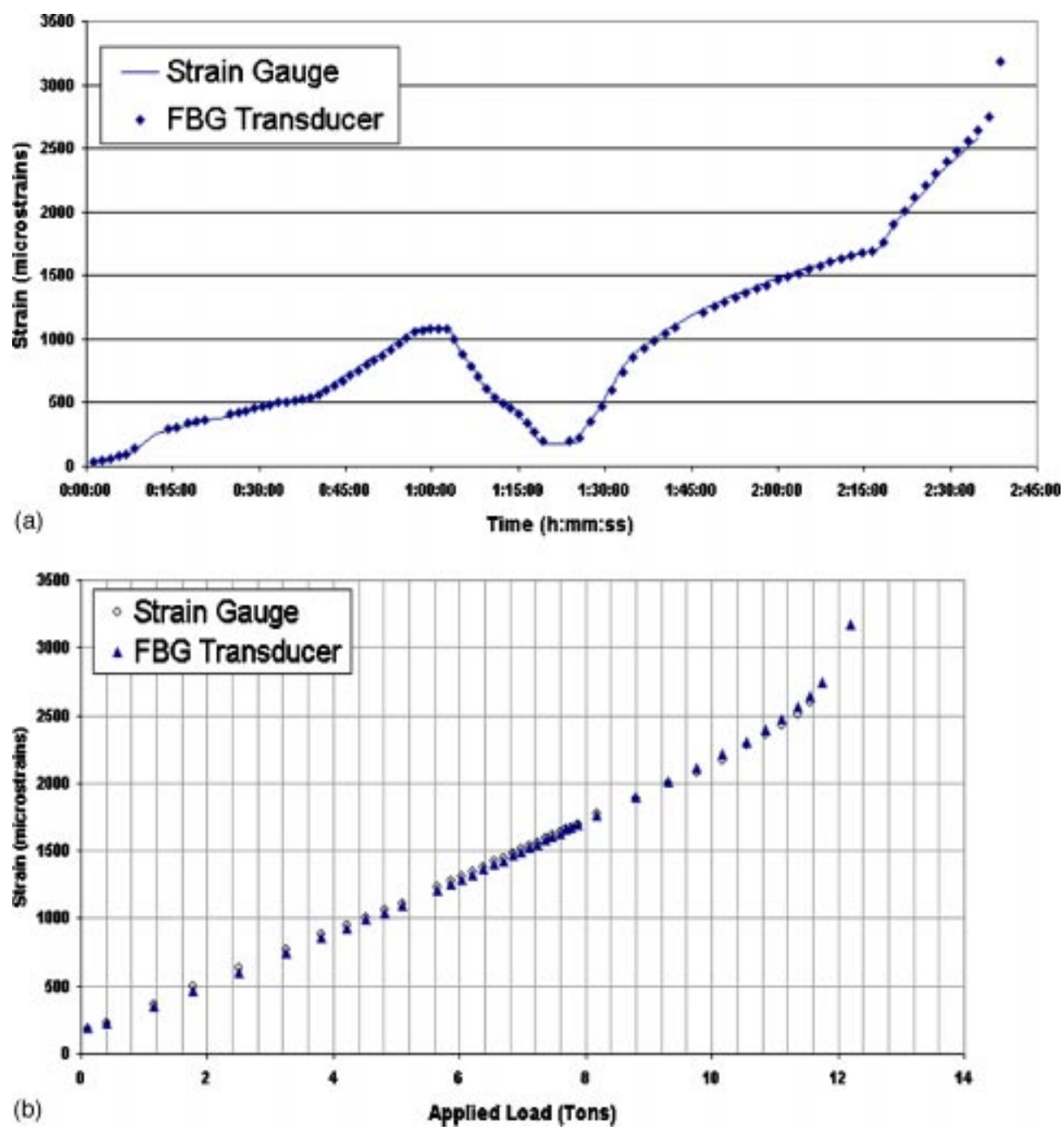

Fig. 6 Response of the gauge-like transducer B to the applied load compared with a conventional strain gauge: (a) strain measured versus time during the load test applied to the beam, and (b) strain versus applied load.

"extreme" regions in which the endurance of the transducers was tested. As a result, it can be concluded that the FBG transducer was able to withstand higher strains than the conventional strain gauge before it broke.

\section{In-Field Demonstrations}

The installations of two different civil structure monitoring systems are described in this section. The first one can be regarded as a test field installation aimed to discover the problems that can arise during this process. It also had the important secondary goal of checking the actual performance of the embeddable transducers that were described in the previous section. This test installation comprised just six transducers. The second one was a much bigger installation that involved a very high number of transducers to monitor a complex structure.

\subsection{Test Installation}

This installation was carried out in a bridge crossing the Autovía del Cantábrico at its kilometric point 48, near $\mathrm{Ca}$ bezón de la Sal, Cantabria, Spain. This structure is a relatively simple one and was chosen because its behavior can easily be theoretically modeled. This allows the comparison of the simulated results with those inferred through the measurements obtained by the photonic transducers. As it has already been said, the installation comprised six transducers distributed in two optical channels. These transducers were installed inside the structure, as described in Fig. 7.

The transducers, all of them of the embeddable type, were installed in key points of the structure. For example, transducers $\mathrm{U}$ and $\mathrm{L}$ (shown in the upper inset of the figure) measured the global bending of the bridge. The transducer at the bottom of the pile accounted for the load transference between the structure and its supporting point. The remaining three transducers, shown in the lower inset of Fig. 7, revealed local behaviors in crucial structural points (the joint between the pile and the body of the bridge, the longitudinal axis of the structure, and one of its wings).

As these were embeddable transducers, their installation had to be carried out in the early days of the construction of the civil structure, when it was still in its iron-rod armor stage. At this early point, prior to the pouring of the concrete, the transducers were attached to the iron skeleton of the structure in a way that they remained unaffected by its deformation. This was done by using turnbuckles. 


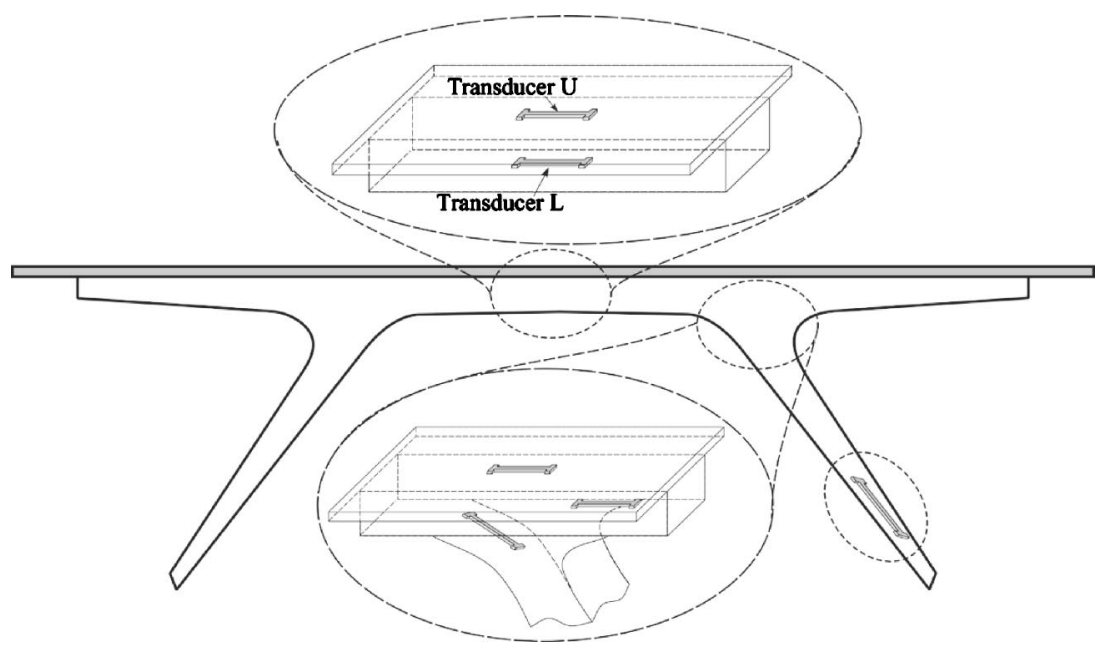

Fig. 7 Distribution of embedded transducers inside the structure that hosts the test installation.

The main goal of this installation was to check whether the transducers were able to withstand the hard installation process and the construction of the structure. On the other hand, a secondary goal was to carry out a comparison between this technology and traditional techniques. On account of the first aim, it can be said that the whole set of transducers survived both processes. On the other hand, to accomplish the desired comparison between photonic and traditional technologies, a set of mechanical extensometers were installed in the bridge. Thus, the measurements obtained by photonic and traditional means could be compared by carrying out a computer-aided transformation. This transformation was required, since the two set of transducers measure different magnitudes at different points.

As it has already been said, the six transducers were distributed in two branches. This fact forced the use of an optical switch (86062C from Agilent Technology, Palo Alto, CA). Also, a FBG interrogation unit (FBG-IS 35 from Micron Optics, Atlanta, GA) was used for recovering the measured information from the transducers. The whole interrogation setup was controlled by a laptop computer, which also stored the data.

Once the traditional and photonic transducer interrogation setups were ready, the bridge was loaded with several trucks. These were distributed on top of the bridge in several ways, all of them trying to provoke a foreknown deformation of the structure. As an example of the behavior of the structure and the monitoring system, Fig. 8 shows one of the truck distributions on top of the bridge, together with the temporal response of transducers $U$ and $L$. This graph illustrates a logical behavior of the bridge: when loaded, its upper part compressed and its lower part stretched. Thus, transducers U and L exhibited twin-shaped responses except for the fact that one represented compression (U) and the other elongation (L). Also, there was a noticeable difference between the amplitude of both responses. This is because concrete has a different mechanical behavior when compressed or stretched. This way, for achieving the same amount of deformation, a bigger force must be applied when compressing.
Not only does this figure reveal characteristics of the quasistatic behavior of the structure, but also of the dynamic one. For example, some details such as the instants at which the different trucks crossed the locations of the transducers (during the positioning stage of the trucks) can be clearly seen as sharp spikes. Moreover, having a closer look at this stage, it can be seen that a progressive loading of the structure was also detected (represented in the graph as steps, like that around 11:08 a.m.). This stepped loading was a consequence of the truck's gradual entry on the bridge.

There is still another detail about this figure worth being highlighted, and it is the remaining structural deformation seen at the end of the load test. This is shown in the graph by a nonzero return behavior, and was due to some permanent structural damage caused during the test. Nevertheless, this kind of damage, which happens quite often during load

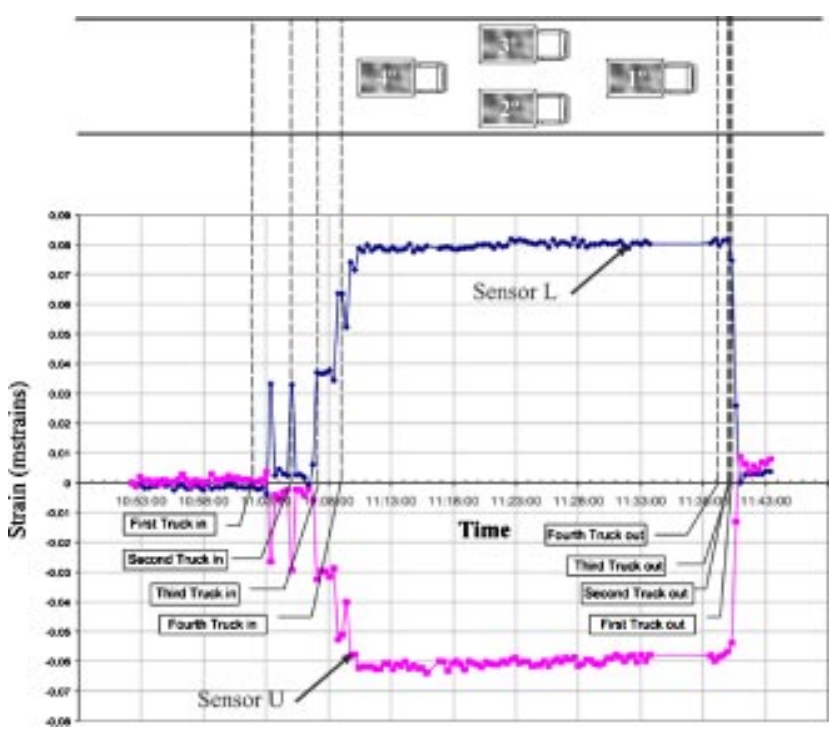

Fig. 8 Truck distribution on the bridge and response of the transducers to the applied deformation. 


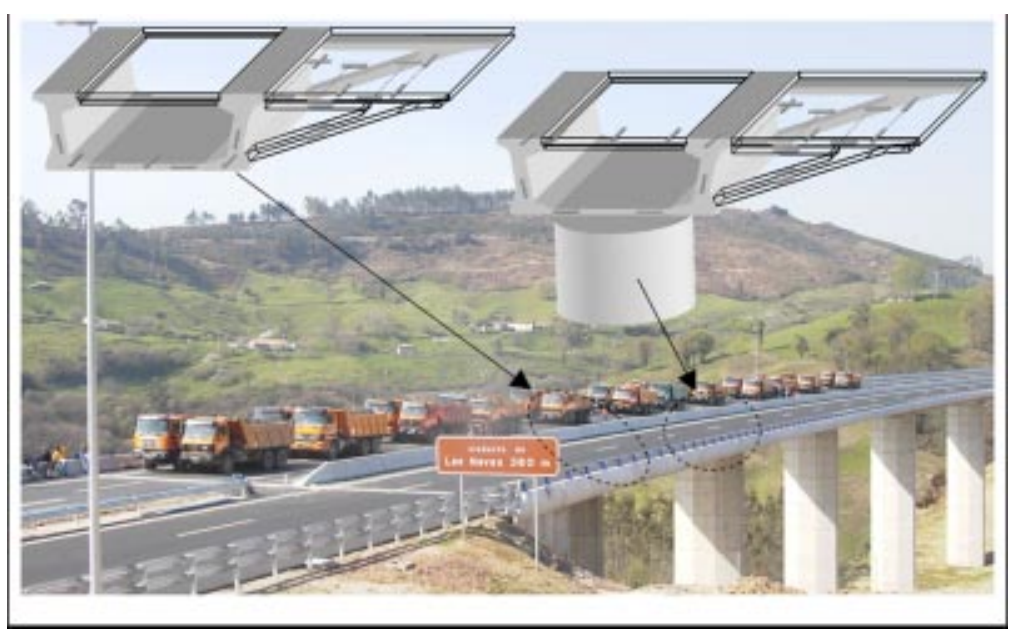

Fig. 9 Photograph of the monitored structure during the load test. The insets show diagrams of the distribution of transducers in two sections of the bridge.

tests, does not compromise the safe operation of the structure. This residual deformation was seen both by the photonic transducers and the mechanical extensometers. Although it is not shown here, the data correlation between these two techniques was fairly good.

It must be said, on behalf of the reliability of the system, that it is still properly working and not a single transducer has been lost.

\subsection{Complex Structure Installation}

After the sensor system was successfully tested on a real civil structure, the next step was to install a similar system in a much more complex structure: a long concrete bridge. Apart from the goal of further testing the monitoring system, it was expected to help civil engineers better understand the behavior of the host structure. That is why it was a much bigger installation than the previous one. This case comprised a very high number of transducers (42) distributed in 15 branches (their distribution can be seen in the insets of Fig. 9). The monitored structure was the Las $\mathrm{Na}$ vas bridge in the Autovía del Cantábrico, a new highway near Cabezón de la Sal, Cantabria, Spain. As this is a symmetric and repetitive structure (it is formed by ten identical sections limited by two piles each), just two sections were monitored: one that rests just on top of one pile and the other in the middle point between two piles. So, as described in Fig. 9, the transducers were installed in key points of the structure, each intended to measure a particular effort.

In installations as big as this one, several precautions must be observed. It is only by experience that these are known. Civil structures bigger than a critical size cannot be built all at once, but they are constructed in phases instead. This means that when a section of the structure is ready for the installation of the transducers, another is being built. In other words, there is a lot of activity around the installation place: lots of people soldering, cutting iron rods, hitting the structure to accommodate different ducts inside the armor, etc. All these multiply the risks of having a failure in the monitoring system caused by a break in the channel (in such an environment, a fiber optic channel is very likely to be cut if no special care is paid), or by excessive attenua- tion (like that introduced by two iron rods pinching the channel), or by direct damage of the body of the transducer. This implies that no matter how well the channel and the transducers are protected, the loss of one or more sensing points is very likely to happen. To minimize these losses and their effects, there are several recommendations that can be followed: first of all, and the most important, the different optical branches should be accessible by their two extremes; secondly, none of these branches should comprise a high number of transducers. The first recommendation increases the reliability of the system, because if the channel is broken in one of its middle points, all the transducers are still accessible either by one extreme or the other. Therefore, no loss of information will take place. The second recommendation complements, in a certain way, the first one, since its importance becomes apparent in those cases in which the former fails. That is, this second recommendation tries to minimize the number of transducers lost in case the channel was broken in two or more points. This recommendation helps to reduce the length of the optical channels. Moreover, because the shorter the channel length the shorter the probability of being damaged, it also helps to increase the global reliability of the system.

Talking about a real interrogation system is talking about something much more complex than just an interrogation unit and an optical switch. In fact, the term "interrogation system" involves the mentioned elements plus complex control software able to identify, in every moment, the transducer that is the origin of the received data. The complexity of such software can be intuited, taking into account that, due to break points in the channel, two transducers of the same optical channel can be interrogated through different ports of the switch. Moreover, fiber breaks can also take place within a transducer. Thus, it can happen that the sensing and reference FBGs of a transducer have to be reached through different ports of the switch. To complicate things further, it is also possible to lose the reference FBG of one transducer. In this situation, its function should be taken over by the reference FBG of the nearest transducer, no matter whether it belongs to the same optical channel or not. 

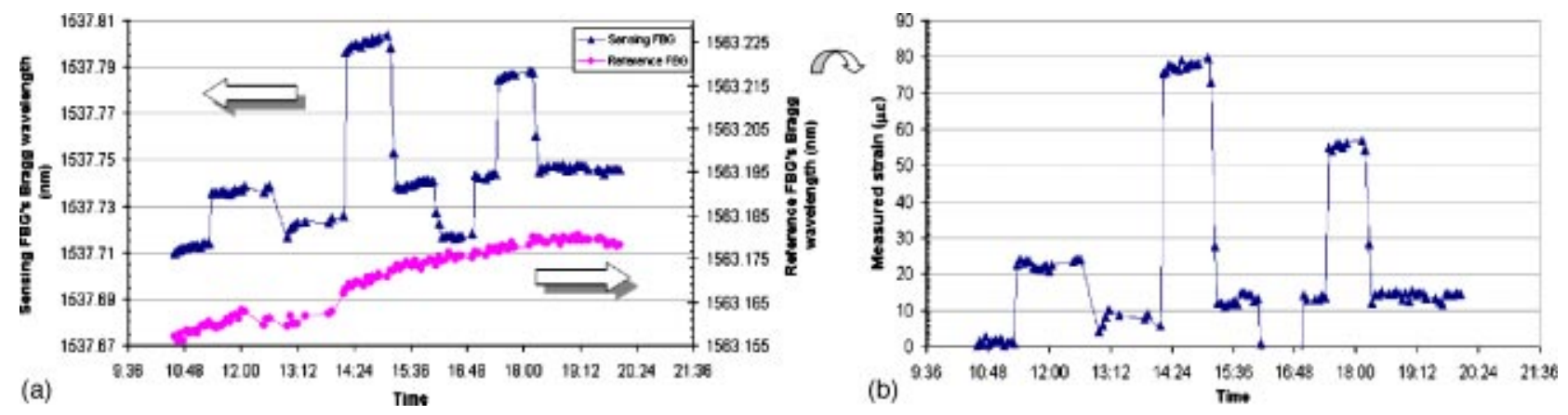

Fig. 10 Response of a transducer to the load test: (a) graph showing the response of the sensing FBG affected by the temperature drift (line with triangles) and the response of the reference FBG (dotted line), and (b) temperature-compensated response of the sensing FBG rescaled to be represented in $\mu \epsilon$.

Homemade software, satisfying all the previous requirements, was developed and successfully tested during the research and development works reported here. This software was able to control both the interrogation unit and the optical switch. It also recorded in a database the measured data. Thus it was possible, among other things, to track the evolution of a single transducer's response, as well as to accomplish the temperature drift compensation in real time.

The temperature compensation topic had a particular importance in this case, since the drifts derived from the FBG cross sensitivity could be similar to, or even bigger than, the actual measurements, as shown in Fig. 10. The response of one transducer to four different quasistatic load tests can be seen [superimposed to the thermal drift in Fig. 10(a), and without that effect in Fig. 10(b)]. This transducer was installed at the central bottom part of the structure in the section between piles. In Fig. 10(a), the upper trace belongs to the strain- (and temperature-) sensitive FBG, while the lower one comes from the reference FBG. The latter clearly shows the temperature evolution inside the structure during a day. The fact that the maximum thermal drift took place around 19:00 instead of around midday is a curious result. There are two main reasons that justify this: the thermal inertia of concrete and the fact that this particular transducer was facing west. As this last figure clearly illustrates, it is mandatory to carry out a strain/temperature discrimination if accurate measurements are to be done.

The quasistatic load tests were meant to check the behavior and mechanical integrity of the structure. It comprised four different situations, called "hypothesis" in technical language. These hypotheses generated all those steplike responses seen here and there in Fig. 10. The first one set 24 loaded trucks right on top of the monitored sections. This situation was maintained for an hour. As can clearly be seen, an elongation of the transducer, and therefore, of the bottom part of the structure, was provoked. It can also be appreciated that, when the trucks were retired from the bridge, it did not return to its original zero level. Instead, there was a residual strain due to the "settlement" of the structure, that is, to some minor structural failure caused by loading. The second hypothesis set the loaded trucks in three groups of 12 on top of alternate sections of the bridge (including the ones instrumented). This situation was maintained over almost an hour. In this case, the response of the transducer was more than twice that due to the previous hypothesis. This was due to the cumulative effect of the loaded-unloaded sections. This way of loading the bridge caused a sinusoidal strain distribution of which amplitude was bigger than the one provoked by placing all the trucks in the whereabouts of the transducer. As in the previous hypothesis, but in a minor degree, there was a shift in the zero-strain return point after loading. The explanation of this shift is similar to that already given in the previous case. The third hypothesis was the complementary of that just described, i.e., there were also alternate loaded and unloaded sections but, in this case, the loaded ones were the previously unloaded and vice versa. As expected, the behavior of the transducer was opposite of the previous cases. In this situation, it was compressed instead of stretched. This was because the monitored section remained unloaded, while the ones next to it were loaded. This situation pushed that section of the structure upward and, thus, made its bottom part compress. At the end of this third hypothesis, the response of the transducer returned to the same zero point of the beginning. The fourth and last hypothesis of the load test set 20 trucks at the farthest end of the structure from the monitored sections. This situation, which lasted a little more than half an hour, caused the last steplike response seen in Fig. 10.

Apart from the quasistatic load test that has already been described, a dynamic one was also carried out. It consisted in a loaded truck crossing the bridge, first at a constant speed of $20 \mathrm{~km} / \mathrm{h}$, and later, backward at $10 \mathrm{~km} / \mathrm{h}$. The response of the transducer is shown in Fig. 11. The two moments at which the truck crossed the monitored area were reflected as two clear increments in the strain. But there are important and interesting differences between these two peaks. The first is that the second peak (corresponding to the backward crossing) was more or less twice as wide as the first one. This was to be expected, since the speed was half that of the forward crossing. The second difference, which is also the most interesting, is the fact that the second increment of strain was double-peaked. These two peaks corresponded to each of the wheel axes of the truck. The first peak was wider than the second one because, as in most trucks, the rear wheel axis was double. This axis detection only took place in the backward crossing, because the interrogation frequency (about $10 \mathrm{~Hz}$ ) was too low for higher speeds. This demonstrates that, using a 


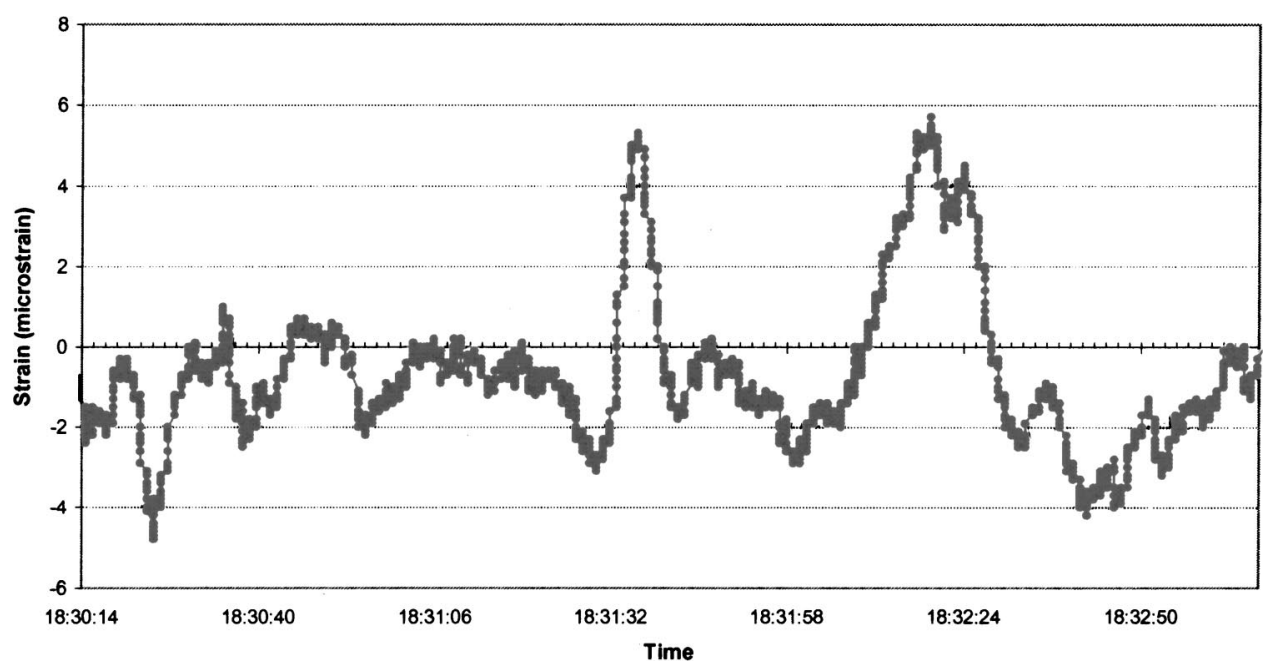

Fig. 11 Dynamic response of a transducer recorded during the crossing of a truck over the monitored area. The truck moved, first forward at $20 \mathrm{~km} / \mathrm{h}$, and later backward at $10 \mathrm{~km} / \mathrm{h}$.

faster interrogation unit, the sensor system will allow dynamic measurements.

Today, more than a year after installation, the system is still properly working.

\section{Conclusion}

The complete process that leads to monitoring a civil engineering structure is described in detail in this work. This description includes steps such as: the design of the transducer, the laboratory demonstrations, field tests, and the real installation.

Two different sets of transducers are designed and successfully tested and installed: one meant to be embedded in concrete and another one thought to replace traditional strain gauges with a minimum technology-change shock. Both kinds of transducers are provided with the elements required to perform strain/temperature discrimination. Therefore, they are able to simultaneously measure these two parameters. Laboratory tests are carried out to check the performance of the transducers in measuring both temperature and strain inside and outside of a concrete beam. The results are satisfactory.

These transducers are also tested in two bridges of a highway in northern Spain, performing very well in both cases. The system demonstrates its ability to simultaneously measure strain and temperature. Its potential capacity to perform dynamic measurements is also tested, casting good results.

To sum up, a complete quasidistributed fiber optic sensor system, designed to monitor typical civil engineering structures, is developed and tested both in laboratory and in real field conditions.

\section{Acknowledgment}

The authors would like to acknowledge the cosupport provided by the European Community through the 1FD970775 SISFOCDETIC project and by the Spanish CICYT through the TIC-2001-0877-C02-01 SuGARUS and the TIC-2001-4503-E projects.

\section{References}

1. Handbook of Optical Fiber Sensing Technology, J. M. López-Higuera, Ed., John Wiley, New York (2002).

2. Optical Fibre Sensors: Applications, Analysis and Future Trends, J. Dakin and B. Culshaw, Eds., Artech House, Boston, MA (1997).

3. D. Inaudi, A. Elamari, L. Pflug, N. Gisin, J. Breguet, and S. Vurpillot, "Low-coherence deformation sensors for the monitoring of civilengineering structures," Sens. Actuators, A 44, 125-130 (1994).

4. D. Inaudi, S. Vurpillot, N. Casanova, and A. Osa-Wyser, "Development and field test of deformation sensors for concrete embedding," Proc. SPIE 2721, 138-148 (1996).

5. A. D. Kersey, M. A. Davis, H. J. Patrick, M. LeBlanc, K. P. Koo, C. G. Askins, M. A. Putnam, and E. J. Frieble, "Fibre grating sensors,' J. Lightwave Technol. 15, 1442-1463 (1997).

6. A. Othonos and K. Kalli, "Fiber Bragg gratings: fundamentals and applications in telecommunication and sensing," Artech House, Boston, MA (1999).

7. J. D. C. Jones and W. N. McPherson, Handbook of Optical Fiber Sensing Technology, Chap. 19, p. 800, John Wiley, New York (2002).

8. M. G. Xu, J. L. Archambault, L. Reekie, and J. P. Dakin, "Discrimination between strain and temperature effects using dual-wavelength fiber grating sensors," Electron. Lett. 30(13), 1085-1087 (1994).

9. G. P. Brady, K. Kalli, D. J. Webb, D. A. Jackson, L. Reekie, and J. L. Archambault, "Simultaneous measurement of strain and temperature using the first- and second-order diffraction wavelength of Bragg gratings," Proc. Inst. Electron. Eng. Optoelecton. 44(3), 156-161 (1997).

10. W. C. Du, X. M. Tao, and H. Y. Tam, "Fiber Bragg grating cavity sensor for simultaneous measurement of strain and temperature," IEEE Photonics Technol. Lett. 11(1), 105-107 (1999).

11. J. Echevarría, A. Quintela, C. Jáuregui, and J. M. López-Higuera, "Uniform fiber Bragg grating first and second order diffraction wavelength experimental characterization for strain-temperature discrimination," IEEE Photonics Technol. Lett. 13(7), 696-698 (2000).

12. M. Song, S. B. Lee, S. S. Choi, and B. Lee, "Simultaneous measurement of temperature and strain using two fiber Bragg gratings embedded in a glass tube," Opt. Fiber Technol. 3, 194-196 (1997).

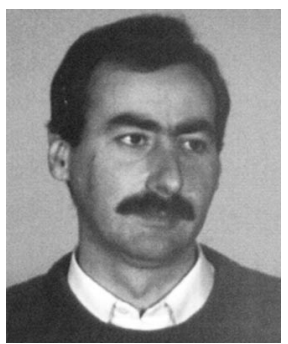

Jose Miguel López-Higuera obtained his telecommunication technical engineering degree from the Universidad Laboral de Alcalá de Henares, and his telecommunication engineering degree in the Universidad Politécnica de Madrid (UPM). He achieved his $\mathrm{PhD}$ degree in telecommunication engineering from the UPM. He founded and is the head of the Photonics Engineering Group of the TEISA Department in the University of Cantabria. Presently, he works on the development of photonics instrumentation, optical fiber sensor systems for civil engineering, electrical power, environmental and smart structures applications, and in optical diagnostics for in- 
dustrial applications. He is senior member of the IEEE and member of the IEE, and OSA.

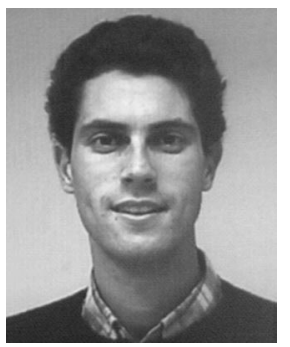

César Jauregui Misas received both his telecommunication technical engineering degree and his telecommunication engineering degree at the University of Cantabria. In 1998 he joined the Photonics Engineering Group. In 2003 he received his $\mathrm{PhD}$ degree. His primary research concern is the interrogation of fiber sensors, especially those based on fiber Bragg gratings and interferometers. He has also developed a research interest for photonic crystal fibers. He is a member of the Optical Society of America (OSA).

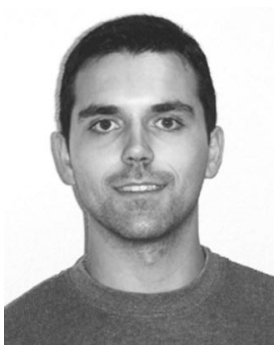

Antonio Quintela Incera received both his telecommunication technical engineering degree and his telecommunication engineering degree at the University of Cantabria. In 1998, he joined the Photonics Engineering Group, where he is currently working toward the $\mathrm{PhD}$ degree. His primary research concern is the writing of fiber Bragg gratings and fabrication of transducers based on FBG.

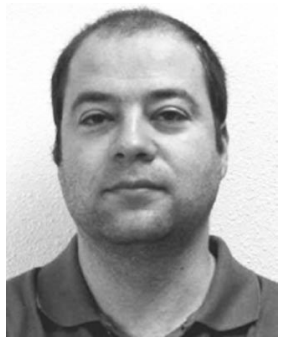

Juan Echevarría Cuenca received the BEng (tech) degree in electrical engineering in 1989 and the BEng (tech) and BSC degrees in telecommunication engineering in 1993 and 1994, respectively, all from the University of Cantabria, Spain. In 2002 he received his $\mathrm{PhD}$ degree in photonics topics. His current research interests include optical sensors, Bragg gratings, and interferometers. 